\title{
Microbiological and Histopathological studies on cholesterol reducing effect of lactobacillus acidophilus
}

\author{
Kamel M. Elmogy, Hanan Hassan and Atef M. Ibrahim*
}

Microbial Biotechnology Departement, Genetic Engineering and Biotechnology Research Institute

(GEBRI), University of Sadat City, Egypt

${ }^{*}$ Corresponding author: atef.ibrahim@gebri.usc.edu.eg

\begin{abstract}
A total of 50 albino rats were used to carry out an in vivo study on the cholesterol lowering impact of isolated $L$. acidophilus isolated from diatery supplements. Blood serum of the experimental rats as well as their liver, heart and aorta tissues were analyzed and examined.The attained results revealed a great decrease in serum cholesterol due to the use of live or dead bacterial cells. The histopathological results collected from examination of liver revealed that in live lactobacillus treated group display significant amelioration compare to non treated group, while no significant improvement detected in dead lactobacillus treated group. The corresponding results of the heart in showed that some pathological lesions seen in cardiac tissue in high cholesterol feeding group, still observed. This is besides atrophy of many cardiomyocytes and apoptosis. Signe of improvement was noticed in dead lactobacillus treated hypercholesterolemic group, where the severity and incidence of the pathological lesions observed in hypercholesterolemic animals was significantly reduced. Concerning the aorta, the attained results demonstrated that in live lactobacillus treated group, early plague formation was observed in few areas, however fibrin covered wide area of the endothelial layer. Significant amelioration in aorta tissues of dead lactobacillus treated hypercholesterolemic group was detected.
\end{abstract}

Key words: diatery supplement, I acidophilus, cholesterol, hupercholesterolem

\section{INTRODUCTION}

The WHO delineated that unhealthy diets such as those high in fat, salt and free sugar, and low in complex carbohydrates, fruits and vegetables, lead to increased risk of cardiovascular diseases (WHO and Organization, 2003). People affected with hypercholesterolemia may avert the use of cholesterol-lowering drugs by practising dietary control or supplementation of probiotics and/or prebiotics. Probiotics are defined as 'living microbial supplements that beneficially affect the host animals by improving its intestinal microbial balances' (Hotel and Cordoba, 2001).

Numerous scintefic papers and reviews articles were published on the health benefits associated with the consumption of the products prepared with probiotic microorganisms (Kehagias et al., 2006, 2006; Lourens-Hattingh and Viljoen, 2001). This is due to the ability of the culture to tolerate acids and bile salts which enable them to implant in 
the intestinal tract (Barnes et al., 2008; Gilliland, 2002). The live cultures of probiotic bacteria in the diet are claimed to provide several therapeutic benefits including disrupt the pathogenesis of hepatic encephalopathy, prevention of cancer, and reduction in the level of serum cholesterol (Barnes et al., 2008; Granato et al., 2010).

Gilliland (Gilliland, 2002), reported that $L$. acidophilus reduces the blood cholesterol by direct breakdown of cholesterol and deconjugation of bile salt. They also examined effects of consumption of one daily serving of yogurt on serum lipids and found a significant reduction in serum cholesterol by performing two controlled clinical studies. Therefore, it can be concluded that since every $1 \%$ reduction in serum cholesterol concentration is associated with an estimated $2 \%$ to $3 \%$ reduction in risk for coronary heart disease, regular intake of fermented milks containing an appropriate strain of $L$. acidophilus has the potential of reducing risk for coronary heart disease by 6 to $10 \%$.

The cholesterol-reducing abilities of six strains of $L$. acidophilus were investigated and it was reported that- in vivo - cholesterol lowering ability was due to the assimilation of cholesterol by $L$. acidophilus cells or/and attachment of cholesterol to the surface of $L$. acidophilus cells (Sarkar, 2003).

Liong and Shah screened eleven strains of lactobacilli and analyzed bile salt deconjugation ability, bile salt hydrolase activity (BSH) and co-precipitation of cholesterol with deconjugated bile. $L$. acidophilus strains had higher deconjugation ability than L. casei strains. Cholesterol coprecipitation with deconjugated bile increased with decreasing $\mathrm{pH}$. L. acidophilus showed highest deconjugation ability and BSH activity towards bile mixtures that resembled the human bile, and may be a promising candidate to exert beneficial bile deconjugation activity in vivo (Liong and Shah, 2005).

\section{MATERIALS AND METHODS}

\section{Samples}

About 80 diatery supplement samples were collected from microbiology laboratory, NODCAR, Cairo. Egypt

\section{Reagents and Chemicals}

MRS agar and MRS broth were obtained from DIFCO Chemicals Company Crystal violet, Safranin, lodine crystals, Potassium iodide, formalin , Ammonium oxalate, haematoxylin and eosin were obtained from Sigma Chemicals Company (St. Louis, Mo, USA), Atorvastatin from South Egypt Drug Industries Com. ( 6 October City Giza Egypt) in the form of tablets .

\section{Animals}

Fifty albino rats weighting about 150-165 g were obtained from Breading Center of National Organization for Drug Control and Research (NODCAR).

\section{Isolation and identification of Lactobacillus}

Ten grams of the diatery supplement product were suspended with $100 \mathrm{ml}$ sterile distilled water, shacked vigorously, then one $\mathrm{ml}$ of suspension was spreading on the surface of sterile plate of MRS agar and incubated at 37 $\pm 2{ }^{\circ} \mathrm{C}$ for $24 \mathrm{~h}$. After incubation, bacterial colonies were picked up and purified by 
streaking on sterile MRS plates and incubated at $37 \pm 2{ }^{\circ} \mathrm{C}$ for $24 \mathrm{~h}$.

\section{Preparation of Lactobacillus Inoculum}

Ten liters of sterile MRS broth medium were inoculated with fresh culture (incubated at $37 \pm 2{ }^{\circ} \mathrm{C}$ for $24 \mathrm{~h}$ ) of purified Lactobacillus isolate and incubated at $37 \pm 2{ }^{\circ} \mathrm{C}$ for $24 \mathrm{~h}$ under anaerobic condition. After growth, the mass of lactobacillus growth was obtained by centrifugation for $15 \mathrm{~min}$ at $1800 \mathrm{xg}$ at room temperature, the mass was washed many times by sterile distilled water and centrifuge under the same previous conditions. obtained lactobacillus mass was divided into 2 portions, one of them was used as live cells, while the other was used in the present study after killing by Autoclaving for $15 \mathrm{~min}$ at $121 \pm 2{ }^{\circ} \mathrm{C}$.

\section{Identification of lactobacilli:}

Identification of isolated lactobacilli were carried out using Biolog AN MicroPlate Identification System (BIOLOG, Inc. 21124 Cabot Blvd, Hayward, CA 94545 USA

\section{Experimental design}

Rats were divided into 5 groups as follows:

Group 1: Normal untreated control group (negative control). This group was fed on a standard diet and supplied with water.

Group 2: hypercholestrolemic group (positive control): Rats were fed on a cholesterol supplemented diet for 8 weeks to induce hypercholesterolemia then continued to receive diet without treatments (Taranto et al., 2000).

Group 3: live lactobacillus - treated hypercholestrolemic group. These rats were fed on a cholesterol supplemented diet for 8 weeks to induce hypercholesterolemia (Taranto et al., 2000), then continued to receive the live lactobacillus cells for further 4 weeks.

Group 4: dead lactobacillus - treated hypercholestrolemic group. Rats were fed on a cholesterol supplemented diet for 8 weeks to induce hypercholesterolemia, then continued to receive the dead lactobacillus cells for further 4 weeks.

Group 5: $\quad$ Atrovastatin - treated hypercholestrolemic group. Rats were fed on a cholesterol supplemented diet for 8 weeks to induce hypercholesterolemia then continued to receive oral atrovastatine at a dose of 0.18 $\mathrm{mg} / \mathrm{Kg}$ body /day ) for another 4 weeks. according to Guerin et al., 2000 (Guerin et al., 2000).

\section{Hypercholesterolemia induction}

The experimental rats (4 groups) were induced to hypercholesterolemia by feeding them with cholesterol enriched diet containing $1 \%$ cholesterol for 3 consecutive months. Cholesterol was dissolved in sheep tail fat and the hypercholesterolemic diet was prepared by mixing each 10 grams cholesterol dissolved in 100 grams sheep tail fat.

\section{Histological examination}

Small pieces of heart, liver and aorta of both control and treated rats were directly collected after sacrifice and transferred into $10 \%$ formaline solution for fixation then treated and examined using routin histological techniques (Bancroft and Gamble, 2008) 


\section{Results}

After $48 \mathrm{~h}$ of incubation under anaerobic from 0.9 to $3 \mathrm{~mm}$ diameter were determined as condition, lactic acid bacteria in MRS agar Gram positive rod shaped, non-spore forming. yielded large white round colonies ranging

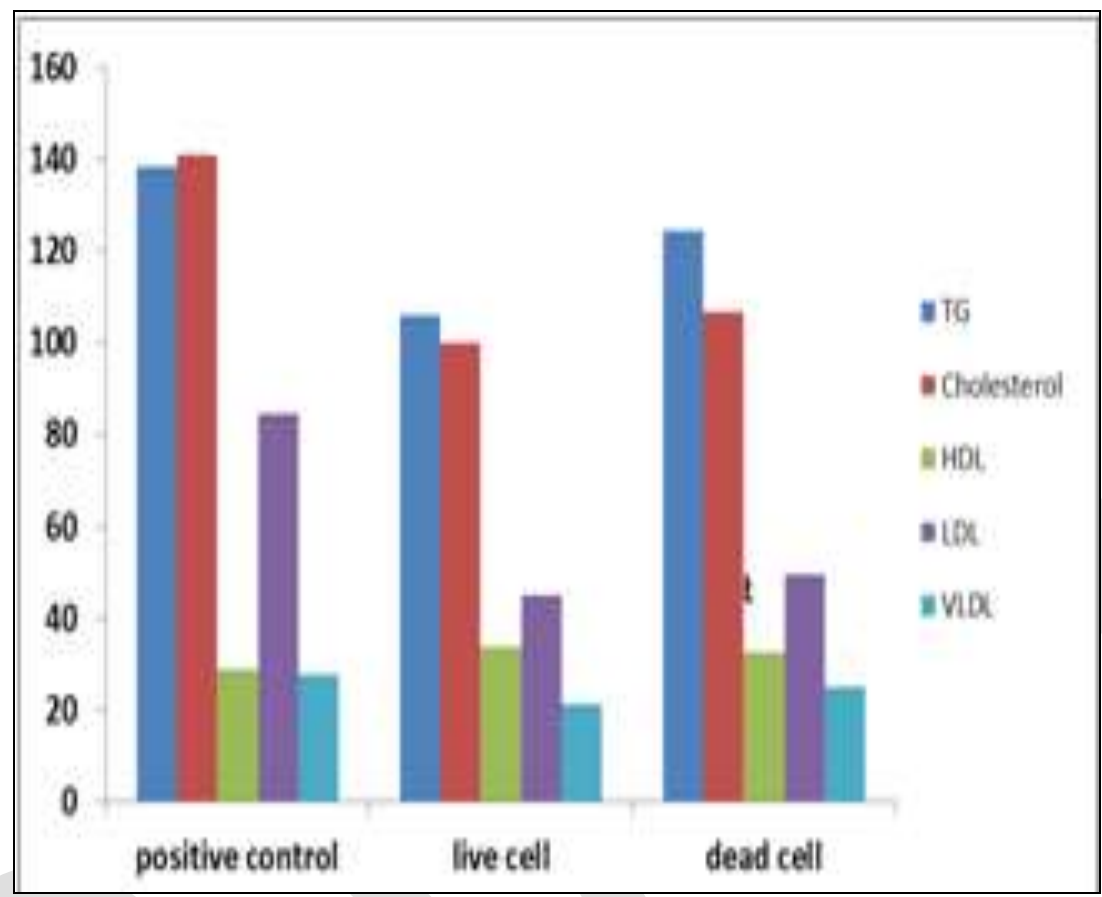

Figure 1: Effect of live and dead L. acidophilus in Blood lipid profil

\section{Biochemical identification:}

Biologs patented redox chemistry makes use of different carbon compounds including sugars, carboxylic acids, amino acids and peptides using Anaerobe Identification Test Panel microplate of Biolog identification system to provide an unparalleled wealth of discriminating biochemical characterizations. A cell suspension resulting metabolic pattern or "fingerprint" generated by the microorganism is recorded and compared to hundreds of identification profiles in a corresponding biolog Database.

Analysis of blood for tiglycerides (TG) content and the different types of cholesterol content as affected by the applied treatments showed that the levels of TG were 138.23,
106.07 and $124.41 \mathrm{mg} / \mathrm{dl}$ for the positive control and for live cells and dead cells treatments respectively. Such results reveal decreasing in TG level by $23.26 \%, 9.99 \%$. for live and dead cells treatments respectevily as shown as in the Fig (1) .

The corresponding values for cholesterol were 141.12, 100.07 and $106.80 \mathrm{mg} / \mathrm{dl}$ in order . these results reveal decreasing in cholesterol level by $29.1 \%$ for live cells treatment and $24.32 \%$ for dead cells treatment relative to positive control .

Concerning LDL, values in $\mathrm{mg} / \mathrm{dl}$ were 84.67, 44.92 and 49.45 for the positive control and treatments of live and dead cells in order, reveals increasing by $46.94 \%, 41.59$ $\%$ for live anad dead cells treatments relative 
to positive control . While HDL showed different trend of resultd since the corresponding values in $\mathrm{mg} / \mathrm{dl}$ were 28.81 , 33.88 and 32.47 for the positive control and treatments of live and dead cells in order, reveals increasing by $17.59 \%, 12.67 \%$ for live anad dead cells treatments relative to positive control. For VLDL content, L.acidophilus live cells decreased the value from $27.64 \mathrm{mg} / \mathrm{dl}$ in positive control to be $21.26 \mathrm{mg} / \mathrm{dl}$ while dead cells decreased the value to $24.88 \mathrm{mg} / \mathrm{dl}$ by by decreasing percentages $23.08 \%$ and $9.99 \%$ for live and dead cells respectively compared to the value recorded for the positive control.

\section{Results of the histological examination:}

\section{Examination of the liver tissues}

Liver tissues of normal control animal (G1) (fig 2), showed the hepatocytes arranged in cords, one or two cells thick, forming the normal liver lobules radiating from the central vein which has extremely thin walls consisting of only endothelial cells .

Microscopic examination of liver tissues of high cholesterol-feeding group ( G2 ) (Fig 3 ), revealed moderate toxicity, in form of congestion and dilatation of portal veins with periportal inflammatory reactions together with proliferated bile ducts. Many central veins showed dilatation and lacerated walls. This is besides dilatation in sinusoids accompanied with proliferated Von Kupffer cells. Hepatocytes display cytoplasmic vacuolation with clear nuclei in some areas or pyknotic ones.Focal necrotic areas with hyaline body could be observed, also, focal area of hepatocytes with fatty degenerative changes.

Mild improvement in liver tissues of atorvastatin treated hypercholesterolemic group (G5) could be seen (Fig 4), where normal liver architecture, normal hepatocytes could be observed. This is besids focal hepatocytes with fatty degenerative changes. However, most of vasculature including portal vein, central Liver tissues in live lactobacillus treated hypercholesterolemic group (G3) displayed great amelioration compare to hypercholes-induced non-treated, where most of the lesions observed were less in severity and incidence. However, few portal areas with mild inflammatory reaction, intact portal vein, this is besides wide areas of intact hepatocytes, while no significant improvement detected in dead lactobacillus treated hypercholesterolemic group (G4), where most of the pathological alteration seen in hypercholesterol-induced non treated animal still was observed (Fig 5) . However, many areas of hepatocytes showed rather normal. 


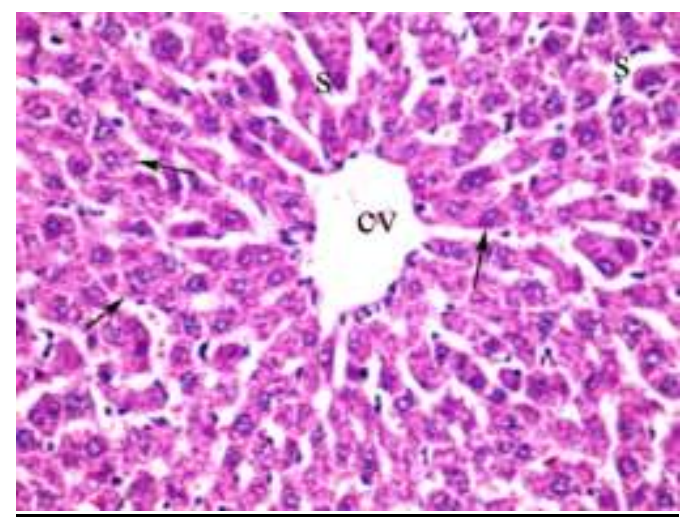

Figure 2: Photomicrograph of liver tissues of control animal ( G1 ) showing normal architecture, central vein (cv), hepatocytes ( arrow), sinusoid(s ). H\&E, X:40

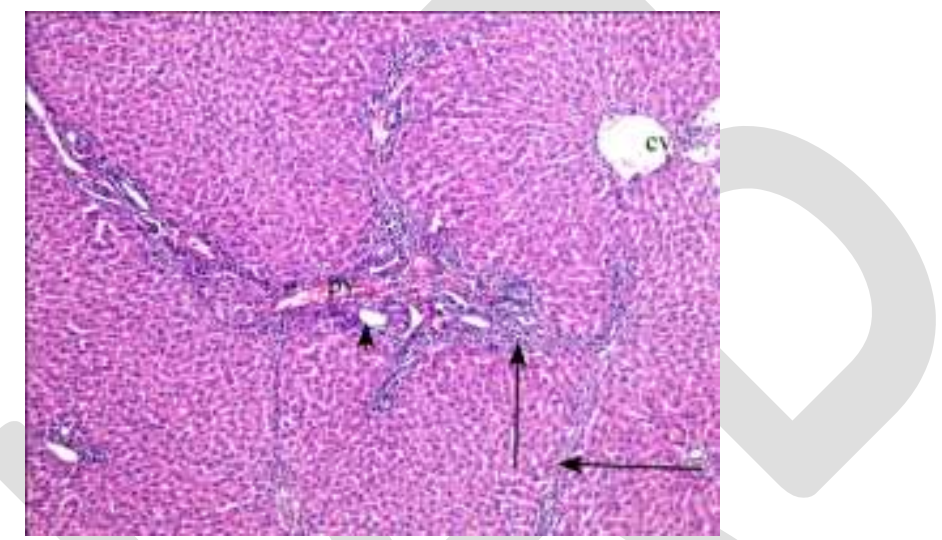

Figure 3: Photomicrograph of liver tissues of high cholesterol diet animals (G2), showing inflammatory reaction (Arrow), central vein (CV), portal vein (PV). H\&E, X: 200

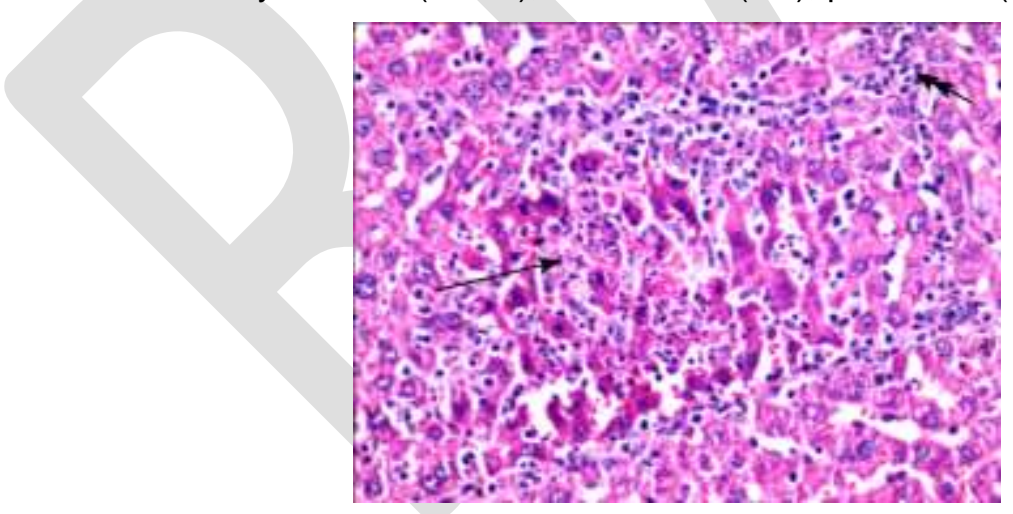

Figure 4: Photomicrograph of liver tissues of atorvastatin treated animal ( (G5), showing Examination of the heart necrotic area (Arrow), inflammatory cells (D.arrow) .H\&E, X: 200. 


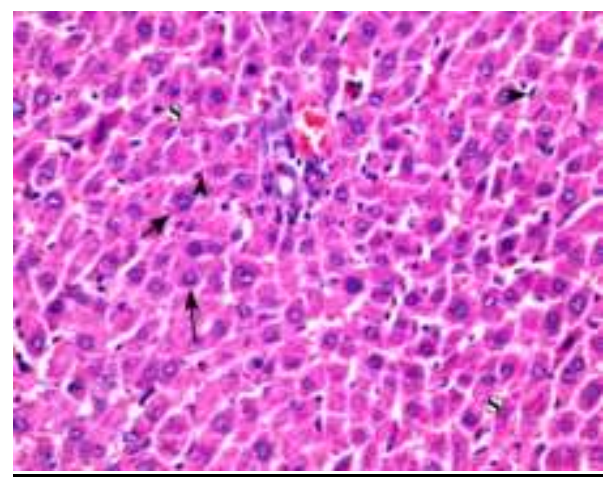

Figure 5: Photomicrograph of liver tissues of Live lactobacillus Treated animal ( G3) showing intact hepatocytes (arrow), proliferated von kupffer cell (arrow head), sinusoids (s). H \& E: X:400.

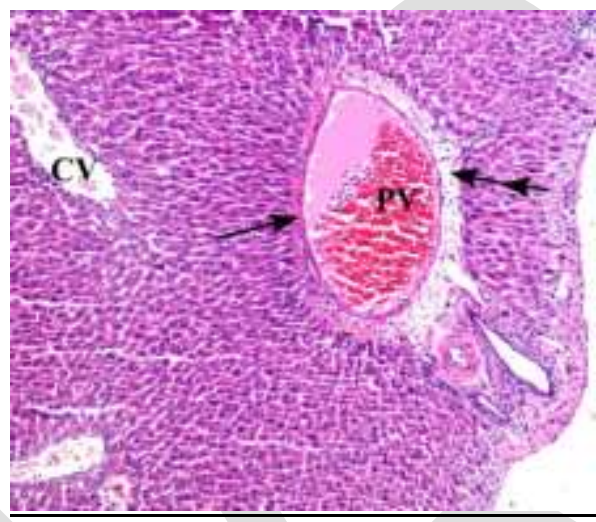

Figure 6: Photomicrograph of liver tissues of dead lactobacillus Treated animal (G4) demonstrating portal vein (PV), peri-vascular edema (double arrow) dilated central vein (CV). H \& E: $x: 100$

Cardiac muscle in the ventricles of control group showed normal histological architecture, where cardiac myocytes exhibited acidophilic sarcoplasm and centrally located nuclei. Blood capillaries appeared in the intercellular spaces. The histological profile of cardiac tissues, of high cholesterol-feeding group (G2) revealed dilated congested vascular channels with sever extravagated RBC'S between cardiomyo fiberlis in many areas (Fig 7 and 8). Degenerative changes in many cardiomyocytes together with separation of myofibrils were detected. Focal area of lipid laden macrophage was noticed. Histological profile of heart tissues of atorvastatin treated hypercholesterolemic group ( G5 ) displayed many intact area in cardiac tissues could be seen, however ,focal area of lipid- laden microphage was observed, in addition to the appearance of many hemorrhagic area in interstitial spaces, cardiac vessels with thickened hyalinized wall, also ,some cardiomyocytes displayed atrophy and homogenous eosinophilic cytoplasm ( Fig 10 ). 


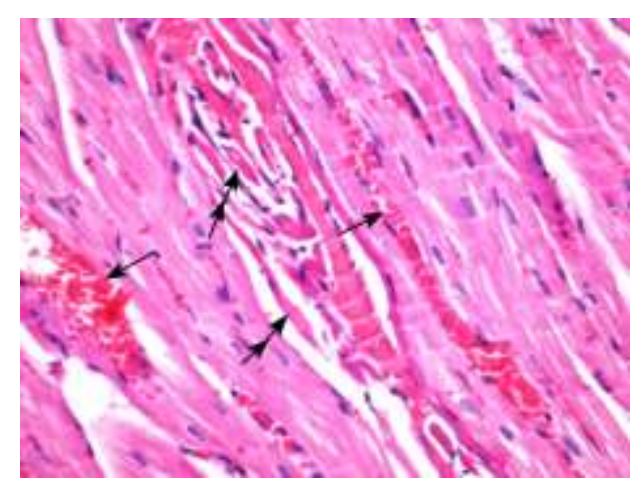

Figure 7: Photomicrograpf of high cholesterol feeding group (G2) demonstrating aggregation of lipid laden macrophage (arrow), cardiomyocytes atrophy (double arrow) with pyknotic nuclei (arrow head). H\& E. 400

In case of live lactobacillus treated hypercholesterolemic group (G3), most of the pathological lesion seen in cardiac tissue in high Cholesterol Feeding group, still observed. This is besides atrophy of many cardiomyocytes and apoptosis, however less inflammatory reaction, with mast cell occasionally seen was detected. Also, many blood vessels displayed collapse,

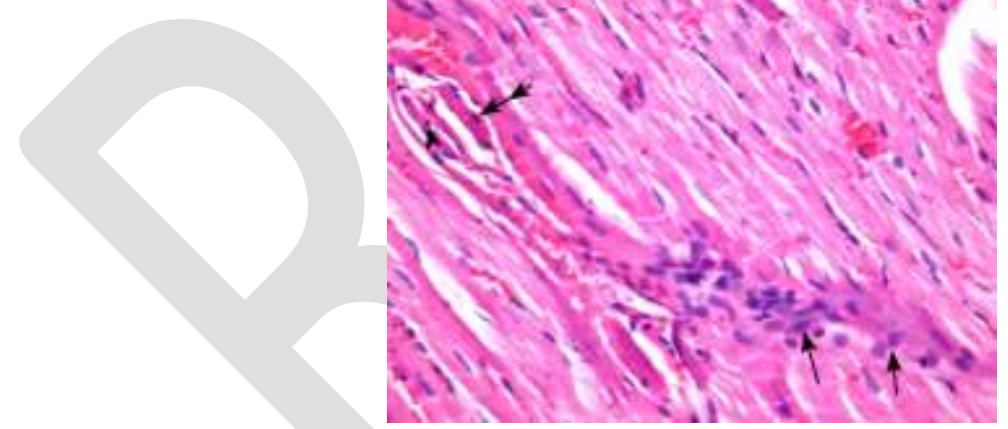

Figure 8: Photomicrograph of cardiac muscle of group 2 showing interstitial hemorrhagic area (arrow), atrophied cardiomyocytes (double arrow). H\&E,X: 400. 


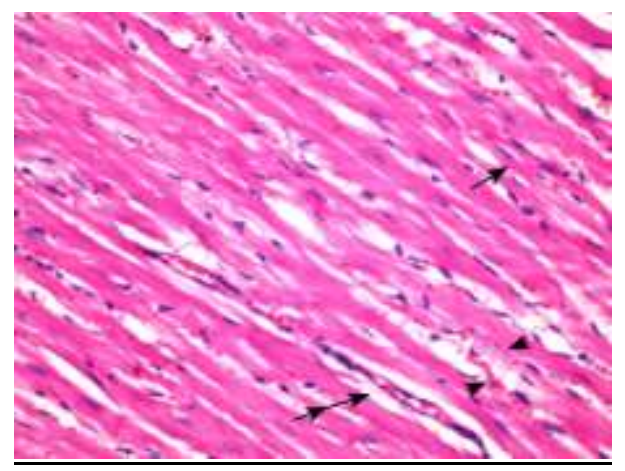

Figure 9: Photomicrograph of cardiac tissues of atorvastatin treated group (G5) showing dilated congested blood capillary(double arrow), together with hemorrhagic area , focal area of cardiomyocytes display atrophy(arrow).H\&E,X:400.

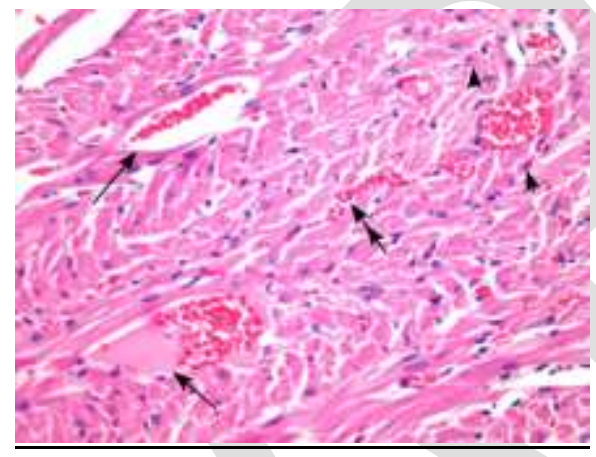

Figure 10: Photomicrograph of cardiac tissues of live and dead-treated groups (G3 , G4) showing dilated congested blood vessels ( arrow), interstitial haemorrage ( double arrow), cardiomyocytes with pyknotic nuclei ( arrow head).H\&E,X: 200.

\section{Examination of the aorta}

Aorta tissues of frank animal displayed three layers called from the luminal side outward, the tunica intima, the tunica media and the tunica adventitia. The tunica intima consists of an endothelium and any subendothelial connective tissue. The tunica media is the layer of concentrically-arranged smooth muscle and contains varying amounts of collagen fibres, elastic fibres, and elastic lamellae. The tunica adventitia is made chiefly of longitudinally arranged collagen fibres. It tends to be much larger in veins than arteries

Photomicrograph of aorta tissues of control group showing normal architecture ( Fig 11 ), while in high cholesterol diet ( Fig 12 ) , many wide areas of intima ( endothelial layer ) were covered with pinkish homogenous layer
, most probably fibrin, together with focal area of vacuolation under endothelial layer of intimae ( early plague accompanied with many homog area of media. Meanwhile in atorvastatin treated hypercholesterolemic group (G5), focal early plaque formation was observed in the intimae (Fig 13).

In live lactobacillus treated hypercholesterolemic group (G3), early plaque formation was observed in few areas, however fibrin covered wide area of the endothelial layer (Fig 14).

Amelioration in aorta tissues of dead lactobacillus treated hypercholesterolemic group (G4) was detected, where focal area of fibrin covering endothelial layer was observed this is beside one plague could be seen (Fig 15). 


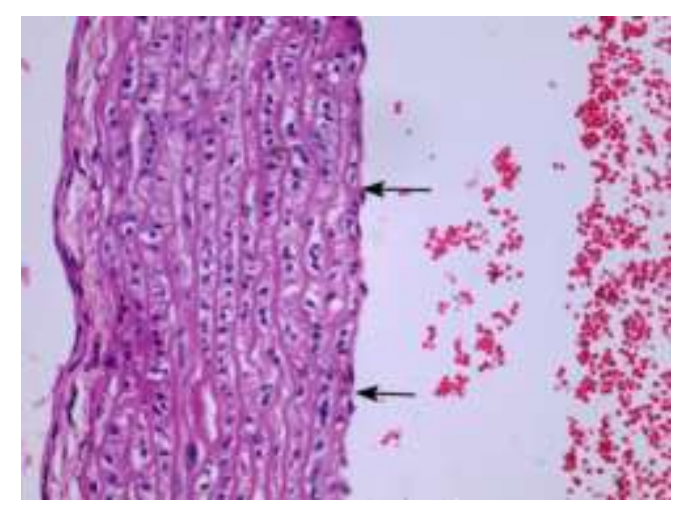

Figure 11: Photomicrograph of aorta tissues of control group (G1) showing normal architecture, endothelial layer (arrow). H\& E, 400.

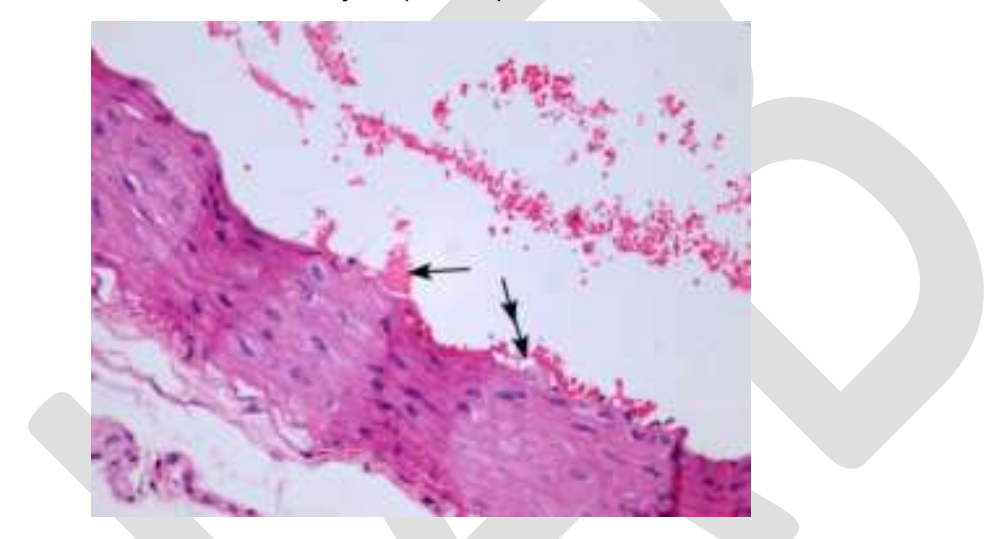

Figure 12: Photomicrograph of aorta tissues of high cholesterol feeding group (G2) demonstrating fibrin deposites (arrow), lipid vacuoles under endothelial layer (double arrow). H \& E, 400.

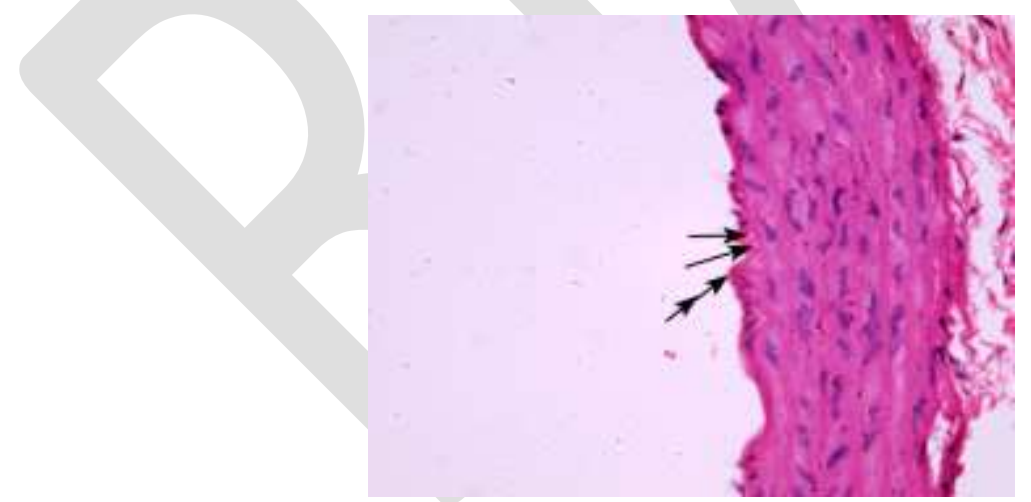

Figure 13: Photomicrograph of aorta tissues of atorvastatin treated group ( G5) showing vacuoles beneth endothelial layer (Arrow), fibrin deposit ( double arrow) ,H\&E,X: 400. 


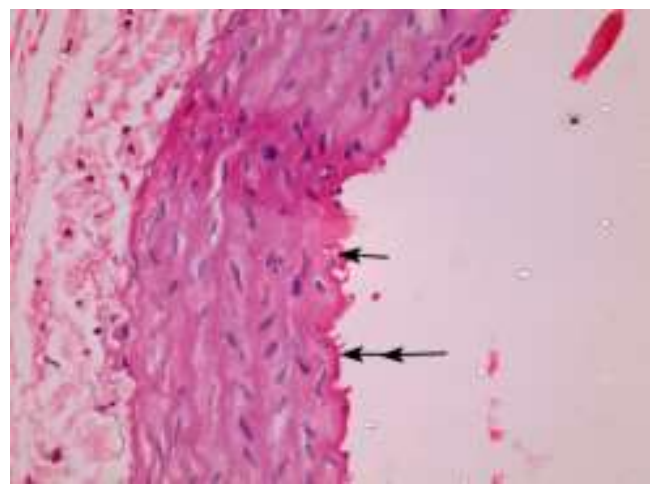

Figure 14: Photomicrograph of aorta tissues of live lactobacillus treated (G3) showing vacuoles beneath endothelial layer, (arrow), fibrin deposits (double arrow). H \& E, 400.

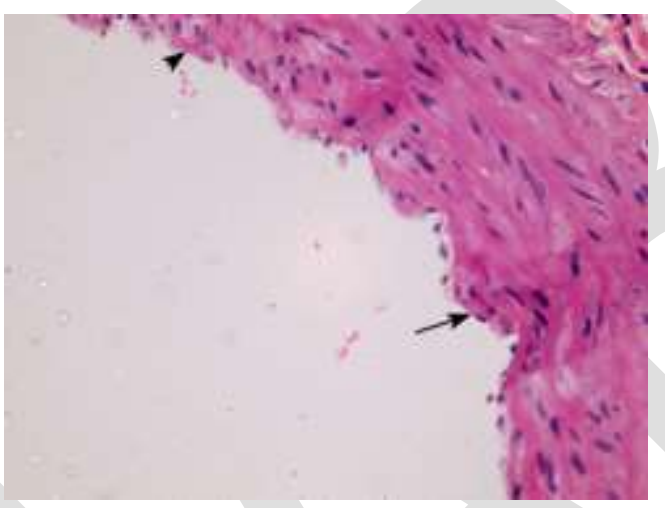

Figure 15: Photomicrograph of aorta tissues of dead lactobacillus treated group (G4) showing intact endothelial layer (arrow), focal area of fibrin (arrow head). H\&E.400

\section{DISCUSSION}

L. acidophilus is the most commonly probiotic used commercially in the production of probiotic fermented milk and pharmacutical diarery supplements. Some researchs (Gilliland, 2002; Lourens-Hattingh and Viljoen, 2001) had indicated that L. acidophilus may provide additional health benefits. Thus, $L$. acidophilus is included in the pharmaceutical supplements which are often recommended after a course of antibiotic therapy

In general, concerning the mechanisms bacteria were given in details in the literature, prevous studies have evaluated a number of mechanisms proposed for the cholesterol-lowering effects of probiotics and prebiotics. One of the suggested mechanisms is the enzymatic deconjugation of bile acids by bile-salt hydrolase of probiotics. Bile, a water- soluble end product of cholesterol in the liver, is stored and concentrated in the gallbladder, and released into the duodenum upon ingestion of food (Gallaher et al., 2000; Kimoto et al., 2002; Lewis and Burmeister, 2005; Liong and Shah, 2005; Park et al., 2007). Cholesterol is used to synthesize new bile acids in a homeostatic response, resulting in lowering of serum cholesterol.

The attained results revealed a great decrease in serum TG level due to the use of live or dead bacterial cells. More decrease was recorded in live cells treatment. This was also noticed on the levels of serum cholesterol, serum LDL and HDL, whereas the live cells caused more increase in serum HDL level as compared to those of dead cells and the 
Apositive controls. It should be taken into consideration the role of the released endoenzymes from the dead bacterial cells in this respect.

Analyses of the blood samples revealed that levels of $T G$, cholesterol, $L D L$ and VLDL were always less in the bacterial treated groups with more decreasing impact due to live-cells treatment. Again, $\mathrm{HDL}$ values were higher in both bacterial cells treatment as compared with HDL in the positive control group.

Our results concerning the lowering action of lactobacillus are in a full agreement with the trends given in the earlier studies carried out in the past and reviewed by Park et al (Gallaher et al., 2000). In a comparison between the bacterial cells and atorvastatin for their cholesterol lowering effect, the attained results revealed nearly the same impact with slightly differences, but the bacterial cells even live or dead and the used atorvastatin showed the same decreasing effect. Thus, the levels of cholesterol as $\mathrm{mg} / \mathrm{dl}$ were $141.12,100.67$, 106.8 and 96.45 for the positive control and treatments of live bacterial cells, dead cells and atorvastatin respectively. This reducing impact was also recorded on the levels of LDL and VLDL, whereas the corresponding values of HDL were 28.81, 33.88, 32.46 and 31.59 $\mathrm{mg} / \mathrm{dl}$ suggesting an increase in the level of the good cholesterol as affected by the applied treatments.

Concerning the histopathological results collected from examination of liver revealed that in live lactobacillus treated hypercholesterolemic group display significant amelioration compared to hypercholesterole -induced non-treated, where most of the lesions observed were less in severity and incidence. Whoever, few portal areas with mild inflammatory reaction, intact portal vein, this is besides wide areas of intact hepatocytes, while no significant improvement detected in dead lactobacillus treated hypercholesterolemic group , where most of the pathological alteration seen in hypercholesterol-induced non treated animal still observed.

The corresponding results of the heart showed that atorvastatin treated hypercholesterolemic group display many intact area in cardiac tissues could be seen , however ,focal area of lipid- laden microphage was observed, in addition to the appearance of many hemorrhagic areas in interstitial spaces, cardiac vessels with thickened hyalinized wall, also ,some cardiomyocytes display atrophy and homogenous eosinophilic cytoplasm. In case of live lactobacillus treated hypercholesterolemic group, most of the pathological lesion seen in cardiac tissue in high cholesterol feeding group, still observed. This is besides atrophy of many cardiomyocytes and apoptosis, however less inflammatory reaction, with mast cell occasionally seen was detected. Also, many blood vessels display collapse. Signe of improvement was noticed in dead lactobacillus treated hypercholesterolemic group, where the severity and incidence of the pathological lesions observed in hypercholesterolemic animals was significantly reduced.

Concerning the aorta, the attained results demonstrated that in live lactobacillus treated hypercholesterolemic group, early plague formation was observed in few areas, and however fibrin covered wide area of the endothelial layer. Significant amelioration in aorta tissues of dead lactobacillus treated 
hypercholesterolemic group was detected, where focal area of fibrin covering endothelial layer was observed this is beside one plague could be seen .

Our data in the present study are in agreement with such finding. Such finding could reveal the trend of our results and the role of lice cells of bacteria in this respect.

The hypocholesterolemic effect of the probiotics has also been attributed to their ability to bind cholesterol in the small intestines. The ability of cholesterol-binding appeared to be growth and strain specific. Kimoto et al. (Kimoto et al., 2002; Lewis and Burmeister, 2005) strengthened such a hypothesis by evaluating the removal of cholesterol by probiotics cells during different growth conditions. Live and growing cells were compared to those that were nongrowing (live but suspended in phosphate buffer) and dead (heat-killed). The authors found that although growing cells removed more cholesterol than dead cells, the heat-killed cells could still remove cholesterol from media, indicating that some cholesterol was bound to the cellular surface. Cholesterol was also removed by probiotics by incorporation into the cellular membranes during growth. They examined the removal of cholesterol by several strains of lactococci from media. The authors observed a difference in the fatty acid distribution pattern for cells grown in the presence and absence of cholesterol. Lipids of probiotics are predominantly found in the membrane, suggesting that cholesterol incorporated into the cellular membrane had altered the fatty acid composition of the cells. The incorporation of cholesterol into the cellular membrane increased the concentration of saturated and unsaturated fatty acids, leading to increased membrane strength and subsequently higher cellular resistance toward lysis.

\section{REFERENCES}

Bancroft, J.D., and Gamble, M. (2008). Theory and practice of histological techniques (Elsevier Health Sciences).

Barnes, P.M., Adams, P.F., and PowellGriner, E. (2008). Advance Data from Vital and Health Statistics; No. 394.

Gallaher, C.M., Munion, J., Hesslink, R., Wise, J., and Gallaher, D.D. (2000). Cholesterol reduction by glucomannan and chitosan is mediated by changes in cholesterol absorption and bile acid and fat excretion in rats. J. Nutr. 130, 27532759.

Gilliland, S.E. (2002). Technological \& Commercial Applications of Lactic Acid Bacteria; Health \& Nutritional Benefits in
Dairy Products. Book Technol. Commer. Appl. Lact. Acid Bact. Health Nutr. Benefits Dairy Prod.

Granato, D., Branco, G.F., Cruz, A.G., Faria, J. de A.F., and Shah, N.P. (2010). Probiotic dairy products as functional foods. Compr. Rev. Food Sci. Food Saf. 9, 455-470.

Guerin, M., Lassel, T.S., Le Goff, W., Farnier, M., and Chapman, M.J. (2000). Action of atorvastatin in combined hyperlipidemia: preferential reduction of cholesteryl ester transfer from HDL to VLDL1 particles. Arterioscler. Thromb. Vasc. Biol. 20, 189-197. 
Hotel, A.C.P., and Cordoba, A. (2001). Health and nutritional properties of probiotics in food including powder milk with live lactic acid bacteria. Prevention 5, 1-34.

Kehagias, C., Koulouris, S., Arkoudelos, J.S., and Samona, A. (2006). Viability and biochemical activity of bifidobacteria in association with yoghurt starter cultures in Bifidus milk and bio-yoghurt during storage at $4^{\wedge}$ oc. Egypt. J. Dairy Sci. 34, 151.

Kimoto, H., Ohmomo, S., and Okamoto, T. (2002). Cholesterol removal from media by lactococci. J. Dairy Sci. 85, 31823188.

Lewis, S.J., and Burmeister, S. (2005). A double-blind placebo-controlled study of the effects of Lactobacillus acidophilus on plasma lipids. Eur. J. Clin. Nutr. 59, 776.

Liong, M.T., and Shah, N.P. (2005). Bile salt deconjugation ability, bile salt hydrolase activity and cholesterol co-precipitation ability of lactobacilli strains. Int. Dairy J. 15, 391-398.

Lourens-Hattingh, A., and Viljoen, B.C. (2001). Yogurt as probiotic carrier food. Int. Dairy J. 11, 1-17.

Park, Y.H., Kim, J.G., Shin, Y.W., Kim, S.H., and Whang, K.Y. (2007). Effect of dietary inclusion of Lactobacillus acidophilus ATCC 43121 on cholesterol metabolism in rats. J. Microbiol. Biotechnol. 17, 655-662.

Sarkar, S. (2003). Potential of acidophilus milk to lower cholesterol. Nutr. Food Sci. 33, 273-277.

Taranto, M.P., Medici, M., Perdigon, G., Holgado, A.R., and Valdez, G.F.
(2000). Effect of Lactobacillus reuteri on the prevention of hypercholesterolemia in mice. J. Dairy Sci. 83, 401-403.

WHO, J., and Organization, W.H. (2003). Diet, nutrition and the prevention of chronic diseases: report of a joint WH.

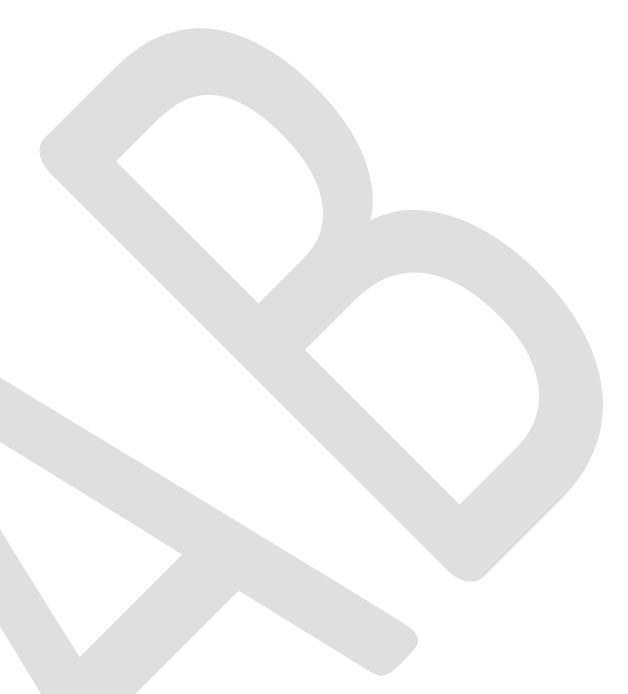

